\title{
Germanica
}

\section{Les hyperboles du narcissisme dans le livret des Stigmatisésde Franz Schreker}

Die Hyperbeln des Narzissmus im Libretto von Franz Schrekers Oper Die Gezeichneten

\section{Alain Leduc}

\section{OpenEdition}

\section{Journals}

Édition électronique

URL : http://journals.openedition.org/germanica/486

DOI : $10.4000 /$ germanica.486

ISSN : 2107-0784

\section{Éditeur}

Université de Lille

\section{Édition imprimée}

Date de publication : 1 décembre 2007

Pagination : 45-61

ISBN : 978-2-913857-20-9

ISSN : 0984-2632

Référence électronique

Alain Leduc, "Les hyperboles du narcissisme dans le livret des Stigmatisésde Franz Schreker », Germanica [En ligne], 41 | 2007, mis en ligne le 01 décembre 2009, consulté le 06 octobre 2020. URL http://journals.openedition.org/germanica/486 ; DOI : https://doi.org/10.4000/germanica.486

Ce document a été généré automatiquement le 6 octobre 2020.

(c) Tous droits réservés 


\section{Les hyperboles du narcissisme dans le livret des Stigmatisésde Franz Schreker}

Die Hyperbeln des Narzissmus im Libretto von Franz Schrekers Oper Die Gezeichneten

Alain Leduc

\section{Résumé de l'opéra'}

\section{Acte I. Une salle du palais d'Alviano Salvago}

1 "L'homme le plus laid de Gênes ", le jeune et riche aristocrate Alviano Salvago, a créé sur une île située en face de la ville un gigantesque parc d'attraction, «l'Élysée », dédié à la beauté : architecture, théâtre, danse, statuaire et art topiaire y concourent à la réalisation d'un spectacle total et permanent réservé à l'aristocratie. Aménagée dans un endroit secret de l'île, une grotte souterraine constitue le chef-d'œuvre d'Alviano, un paradis artificiel où se concentrent tous les moyens susceptibles de provoquer l'ivresse des sens et le désir érotique.

2 Au début de l'opéra, Alviano apprend avec effroi de ses pairs que son île, et en particulier sa grotte mystérieuse, ont été détournées de leur vocation esthétique. Les jeunes aristocrates s'y livrent à des orgies avec des jeunes filles de la bourgeoisie génoise qu'ils font enlever par des sbires.

3 L'indignation d'Alviano fait vite place à l'auto-apitoiement: si ces orgies le révulsent, c'est d'abord parce que sa laideur lui interdit les plaisirs de l'amour physique. Luimême ne s'est plus jamais rendu dans l'île depuis qu'il l'a créée, de peur de souiller sa propre création. À présent, il a décidé d'offrir son œuvre à la ville de Gênes, ce qui provoque la consternation de ses amis. 
4 Tandis qu'Alviano se retire pour préparer la venue du Podestat de Gênes et des sénateurs, à qui il doit faire part officiellement de sa décision, le comte Vitelozzo Tamare, le chef du clan des aristocrates, entre en scène, exalté et à bout de souffle. Il vient de rencontrer une jeune femme dont la beauté l'a bouleversé. C'est alors qu'arrive le Podestat, accompagné de sa fille Carlotta, en qui Tamare reconnaît la belle inconnue. Celui-ci, tandis qu'Alviano s'entretient avec le Podestat, commence sans grand succès à faire la cour à Carlotta. Alviano vient alors convier ses hôtes à prendre part à un banquet.

5 Carlotta, qui a prétexté une indisposition, a quitté le banquet en compagnie d'Alviano. Elle lui explique qu'elle est peintre, que l'âme est son sujet de prédilection et qu'elle aimerait avoir Alviano pour modèle. Celui-ci, qui se croit moqué à cause de sa laideur, a une vive réaction. Carlotta le ramène au calme en lui racontant qu'elle a souvent observé Alviano, depuis son atelier, tandis qu'il se promenait seul dans la campagne. Elle a senti sa beauté intérieure en le voyant, un matin, lever les bras vers le soleil levant. Elle l'a peint dans cette attitude, mais il lui manque encore les traits de son visage. À la fois ému et confus, Alviano accepte de poser pour elle.

\section{Acte II}

\section{Premier tableau. Grande salle dans le palais du duc Adorno}

6 Le Podestat et quelques sénateurs viennent de s'entretenir avec le duc Adorno à propos du projet de donation d'Alviano. Le duc leur a fait une réponse dilatoire qui provoque leur irritation.

7 Le duc fait son entrée en compagnie de son ami Tamare. Celui-ci lui confesse sa passion pour la fille du Podestat et raconte qu'elle a repoussé ses avances et sa demande en mariage le matin même. Le duc propose d'intercéder en sa faveur auprès de Carlotta, tout en préparant son ami à l'éventualité d'un échec. Tamare, à qui aucune femme ne s'est refusée jusqu'à présent, réplique qu'il la prendra de force si elle refuse d'être sa femme. Il en vient alors à révéler au duc le secret de la grotte et des enlèvements et demande, au nom de ses complices, qu'Adorno s'oppose au transfert de l'île, afin de les protéger de la justice. Adorno condamne ces agissements, mais accepte néanmoins, par amitié pour Tamare et pour éviter un scandale. Il quitte la scène en proférant une ultime mise en garde: si Alviano outrepasse son veto, lui et les autres "criminels » n'auront plus aucun soutien et devront répondre de leurs actes.

\section{Deuxième tableau. L'atelier de Carlotta}

8 Tandis qu'Alviano pose pour Carlotta, celle-ci lui parle d'une amie d'antan qui ne peignait que des mains. La plus étrange de ses toiles représentait une main pâle dont les doigts longs et fins serraient un objet invisible dont émanait une lueur rougeoyante. Carlotta explique que cette amie devait renoncer au plaisir de l'amour du fait que son cœur malade n'aurait pu survivre à l'extase amoureuse. La main du tableau représentait la douleur lancinante qui saisissait son cœur dans les moments de grande excitation.

9 La discussion quitte peu à peu le ton badin pour aboutir à la déclaration d'amour de Carlotta à Alviano. Alors que celui-ci est submergé par la passion, Carlotta lui demande de reprendre la pose et, ayant obtenu l'expression souhaitée dans ses yeux, achève le 
tableau en quelques instants. L'émotion qui la saisit lui fait porter la main à son cœur ; elle chancelle et, par inadvertance, découvre un tableau jusqu'ici dissimulé : il s'agit de la main décrite précédemment. Alviano comprend que Carlotta parlait d'elle-même. Il se précipite pour la soutenir, mais, alors que Carlotta s'offre à lui, il contient son ardeur et se contente de l'étreindre chastement.

10 Une servante annonce alors la venue du duc Adorno. Carlotta, étonnée de cette visite, se prépare à le recevoir.

\section{Acte III}

\section{Premier tableau. Sur l'Élysée}

11 Alviano a décidé d'ouvrir son île au public sans attendre la décision du duc. Le peuple génois découvre, médusé, les splendeurs de l'Élysée. Sur ce fond de spectacle nocturne, plusieurs scènes se succèdent.

12 Alviano et le Podestat discutent du transfert de l'île. Alviano espère que ce don pourra racheter les mauvaises conséquences que sa création a entraînées et que le Podestat connaîtra bientôt (Alviano pense à la grotte et aux enlèvements). L'amour de Carlotta lui apparaît comme une récompense et un encouragement à braver la volonté du duc Adorno.

13 Carlotta, maintenant fiancée à Alviano, est en conversation avec le duc. Elle lui avoue que son amour pour Alviano n'a pas résisté à la beauté de l'Élysée. Son fiancé lui est apparu " comme une horrible vermine dans un jardin rempli des plus belles fleurs", dit-elle en reprenant les propres paroles d'Alviano au deuxième acte. Le duc essaie de persuader Carlotta qu'il est rongé par le vice, mais la jeune fille ne veut pas l'écouter. Elle est peu à peu gagnée par la beauté de l'île et n'a plus qu'un désir : retrouver le beau Tamare.

14 Les scènes suivantes décrivent la montée de l'extase collective et, en parallèle, le désarroi croissant d'Alviano qui ne trouve plus sa fiancée. Celle-ci est désormais en compagnie de Tamare qui l'emmène vers la grotte.

15 Au moment où le peuple en liesse acclame Alviano, le Capitaine de la justice, suivi des "Huit », entre en scène et accuse publiquement Alviano, au nom du duc Adorno, d'avoir organisé les enlèvements. Alviano, de son côté, n'entend rien, il est rongé par un terrible pressentiment et croit entendre les voix entremêlées de Tamare et Carlotta. Quand on confirme que Carlotta a disparu, il décide d'emmener tout le monde à la grotte.

\section{Deuxième tableau. Dans la grotte}

Le décor évoque un combat et une orgie interrompue. Tamare et les jeunes aristocrates sont enchaînés, des jeunes femmes se pelotonnent dans des alcôves. Carlotta gît sans connaissance sur un lit couvert de pétales de roses. Alviano interroge Tamare; il refuse de croire que Carlotta se soit donnée à lui de son plein gré. Quand Tamare, plein de sarcasme, lui apprend qu'ils se sont donnés l'un à l'autre en connaissant la conséquence fatale de leur union, Alviano le poignarde.

17 Carlotta se réveille au cri de Tamare. Alviano se précipite auprès d'elle, mais elle le repousse avec horreur et réclame son amant Tamare, avant de rendre l'âme. Alviano 
est gagné par la folie, bute sur le corps de Tamare et s'éloigne sous les regards effrayés de la foule.

Sous sa complexité apparente, l'intrigue des Stigmatisés révèle une structure dramatique assez simple : c'est l'histoire d'une rivalité destructrice entre deux hommes qui aiment une même femme. Schreker a recours dans son opéra au même schéma triangulaire que ses contemporains Zemlinsky dans Une Tragédie florentine (1917) et Berg dans Wozzeck (1923). Le parallélisme avec Berg va encore plus loin dans la mesure où Les Stigmatisés comme Wozzeck adoptent le découpage en trois actes selon le schéma canonique exposition / péripétie / catastrophe. À l'acte I, les deux rivaux font la rencontre de la femme fatale; au deuxième acte, elle semble se décider pour l'un d'entre eux ; au troisième, elle se donne à l'autre, ce qui cause sa mort, celle de l'amant et la folie du rival éconduit. Comparée à celle du Son lointain, la facture dramatique des Stigmatisés est donc beaucoup plus unitaire: aucun saut dans le temps, aucune évocation du passé, aucun dédoublement de l'action ne vient contrarier la progression du drame jusqu'au désastre final. Schreker semble, via la Renaissance qui sert de toile de fond à son opéra, vouloir renouer avec une conception plus classique du drame musical.

19 Et pourtant, la sobriété et l'équilibre de l'intrigue ne constituent qu'un des deux pôles de l'opéra. L'autre est celui de la démesure. Elle apparaît déjà dans le décor et la musique, pareillement fastueux, mais bien plus encore dans la psychologie des trois principaux protagonistes du drame. Alviano Salvago, Carlotta Nardi et Andrae Vitelozzo Tamare sont tous trois des êtres hors norme et, pour bien souligner cet aspect, Schreker a introduit dans son livret plusieurs données peu réalistes, mais qui doivent être acceptées par le lecteur/auditeur, sous peine de rester définitivement à l'extérieur du drame. Ces données sont au nombre de trois.

20 La première est l'Élysée, une île fabuleuse, située dans le golfe de Gênes, où le héros de l'opéra, Alviano Salvago, a créé une sorte de Disneyland pour adultes. Réservée à l'élite aristocratique génoise (avant qu'Alviano décide de l'ouvrir au peuple), cette installation théâtrale suppose une organisation et des moyens gigantesques qui outrepassent largement les moyens d'une ville moyenne de la Renaissance italienne.

21 La seconde est le mystère qui entoure la disparition des jeunes filles de la bourgeoisie génoise. L'idée théâtrale de Schreker se situe à mi-chemin entre Sade et Pasolini, ce qui au fond n'a rien d'étonnant dans la Vienne de Freud et de Schnitzler. Afin d'assouvir leurs appétits sexuels, les nobles de Gênes font enlever ces jeunes femmes et les font participer à des orgies dans une grotte secrète de l'île en question. Le sort réservé à ces femmes reste vague. Certains commentateurs déduisent du fait qu'elles ne sont pas retrouvées que les nobles les mettent à mort. Mais cette supposition ne nous semble pas fondée, car les paroles des aristocrates au premier acte laissent clairement entendre que les jeunes filles finissent elles aussi par tomber sous le charme lénifiant de la grotte et par devenir des victimes consentantes. On peut alors penser que la honte, l'intériorisation du tabou social, les empêchent ensuite de dénoncer leurs ravisseurs. Car les rapts durent depuis des semaines et l'on ne peut que s'étonner que personne n'en vienne à faire peser le moindre soupçon sur les jeunes aristocrates. Ici encore, la réalité fantasmatique prime sur la vraisemblance.

22 Latroisième de ces données est la maladie cardiaque dont souffre Carlotta. L'idée, médicalement peu crédible, que développe Schreker est que Carlotta mourra à sa première relation sexuelle. Le plus étonnant est que Carlotta exclut elle-même 
délibérément toute autre possibilité d'avoir une crise cardiaque. Elle associe d'emblée dans son psychisme l'amour et la mort.

À travers ces trois «licences » théâtrales: l'île, les enlèvements et la maladie de Carlotta, on voit bien que l'intrigue a essentiellement pour but de permettre à Schreker d'explorer les tréfonds de la psyché humaine. Les trois données invraisemblables ont une fonction de verre grossissant; elles incarnent, avec une démesure à la fois théâtrale et démonstrative, les obsessions et les fantasmes pulsionnels qui habitent chacun des trois personnages principaux de l'opéra. L'improbable Élysée est ainsi le fantasme compensatoire d'Alviano; les enlèvements sont l'incarnation de l'égoïsme dominateur et amoral qui caractérise Tamare ; la maladie de cœur, enfin, est la fusion complète d'Éros et de Thanatos dans la psyché de Carlotta. Le point d'intersection de ces trois hyperboles est clairement la pulsion sexuelle: objet de sublimation chez Alviano et d'idéalisation chez Carlotta, moyen de réalisation de soi chez Tamare. Mais le propos de l'opéra ne s'arrête pas là ; il ne s'agit pas seulement pour Schreker de mettre en scène trois manières de vivre et éventuellement de réaliser ladite pulsion, mais d'indiquer surtout ce qui vient la pervertir. À travers leur valeur hyperbolique, les trois données invraisemblables désignent le narcissisme commun aux trois personnages comme la cause première du désastre absolu qui clôt l'opéra.

\section{Alviano, Narcisse de l'image}

Figure centrale de l'opéra, Alviano Salvago est d'abord présenté comme le créateur de l'Élysée, ce gigantesque complexe tout entier dédié aux plaisirs des sens qu'il a fait construire sur une île au large de Gênes. Au cours de la discussion avec les nobles génois qui ouvre l'opéra, Alviano ne fait pas mystère des raisons qui l'ont poussé à créer l'Élysée. C'est pour compenser les défauts de son apparence physique - Alviano est petit et contrefait - qu'il a imaginé et puis réalisé cette œuvre démesurée, bien plus proche de l'actuel parc d'attractions que des spectacles en plein air historiquement attestés au temps de la Renaissance.

Mais l'Élysée n'est pas que la réalisation d'un fantasme compensatoire; il est tout autant le résultat d'une projection narcissique, ce qu'un examen du livret révèle rapidement. Alviano est maladivement obsédé par son image et c'est précisément pour se construire une image de substitution qu'il a créé l'Élysée. Cette œuvre consacrée au culte de la belle apparence - ce qui au passage lui barre l'accès au statut d'œuvre d'art véritable - fonctionne dans sa sensualité exacerbée bien moins comme porte-parole de l'esprit d'Alviano que comme un substitut de son propre corps lui permettant d'accomplir par procuration ses fantasmes de séduction. La très longue didascalie qui ouvre le troisième acte montre que le créateur de l'Élysée a soigneusement établi le programme des festivités qui attendent le public. Il s'agit de séduire celui-ci en le soumettant à différents types d'impressions sensorielles et de le faire passer progressivement d'un état d'excitation sensuelle assez anodine à la lascivité la plus débridée. L'aboutissement implicite de ce parcours initiatique étant la grotte secrète où en toute logique la tension érotique accumulée est appelée à se libérer dans l'acte sexuel.

On l'aura compris, l'Élysée n'est rien d'autre qu'une allégorie du corps séduisant qu'Alviano aurait aimé posséder et de la séduction qu'il aurait aimé exercer à travers lui, la projection narcissique et idéalisée de son moi de surface. De cette manière, on 
s'explique mieux l'empressement d'Alviano à vouloir offrir son île à la ville de Gênes. L'esprit même de l'Élysée étant la séduction, il va de soi qu'il ne pouvait rester dans la confidentialité. Alviano veut maintenant tirer un minimum de profit de cette véritable machine à séduire dans laquelle il a investi son temps, son énergie créatrice et englouti la moitié de sa fortune. Ce n'est d'ailleurs pas sans une certaine solennité qu'il en fait part à ses compagnons, comme s'il s'agissait d'un don de soi : «Demain soir, après la tombée de la nuit, en présence du Haut Sénat et du Podestat, en grande pompe, je la livrerai au peuple entier. Messieurs, vous êtes invités².»

$\mathrm{Au}$ troisième acte, l'amour de Carlotta - qu'il considère désormais comme sa fiancée amène Alviano à donner un sens différent à sa donation. Celle-ci était initialement de nature narcissique : en offrant l'Élysée à la ville, Alviano voulait s'offrir lui-même à l'admiration du public, en compensation de son image défectueuse. Maintenant qu'il croit posséder l'amour de Carlotta, l'île a perdu à ses yeux son urgence émotionnelle ; elle n'est plus qu'un objet esthétique auquel il va jusqu'à confier, lors de sa conversation avec le Podestat, une valeur morale :

Alviano

[...] Mais j'étais conscient de m'être rendu coupable d'une idée sacrilège, dont je maudissais les fruits et dont le venin empoisonnait mon âme. Pour expier, j'ai sacrifié ce qui était à l'époque le plus cher à mon cœur, le seul bonheur de mes jours sans joie, sans le regretter, car j'en ai tiré une récompense imméritée 3 .

Ces paroles remplies de bons sentiments et de confiance en soi vont en quelque sorte fixer la hauteur de la chute que le jeune aristocrate va accomplir jusqu'à la fin de l'opéra. Conjuguée à la fièvre érotique qui s'empare progressivement de la foule, l'absence prolongée de Carlotta finit par plonger Alviano dans l'angoisse. Celui-ci sent confusément que son bonheur était sans doute trop rapide et trop parfait pour ne pas être une illusion. La figure de Carlotta retrouve peu à peu, dans son esprit, son statut d'idéal inaccessible et de fantasme :

Alviano (comme oppressé)

J'entends sa voix! Elle me parvient des hauteurs par-delà les précipices en mille échos! Mélodie aérienne, chanson railleuse émanant des tréfonds de l'enfer! Son image danse devant moi, émerge furtivement des buissons et des haies, çà et là je vois scintiller sa robe, elle me poursuit comme un feu follet, dans l'éther, dans les marais ; pourtant je la tenais, elle était mienne ! Ou peut-être pas ${ }^{4}$ ?

Alors que le peuple porte son bienfaiteur en triomphe, Alviano se sent misérable. En perdant l'amour de Carlotta qui le conduisait au-delà de lui-même, il redevient la proie d'un narcissisme d'autant plus acerbe que l'Élysée a perdu à ses yeux toute sa valeur compensatoire. La grotte souterraine où se déroule l'ultime scène de l'opéra fournit le symbole topographique de cet enfermement narcissique. Alors que Carlotta gît, mourante "sur un lit de pétales de roses ", l'unique préoccupation d'Alviano est de savoir de la bouche de son rival si la jeune femme s'est donnée à lui de plein gré ou si elle a été violée. Dans le dernier cas, Alviano pourra se raccrocher à l'image de soi qu'il s'était forgée grâce à l'amour de Carlotta : "Mais ici au fond de moi subsistera quelque chose, comprends-moi, comme une triste consolation. ${ }^{5}$ » Dans l'autre cas, c'est une chute sans fin qui attend Alviano:

Mais si tu me dis : "Elle s'est donnée à moi, elle, Carlotta, librement par amour et elle était heureuse ", oui, vraiment, tu as dit « heureuse », alors je n'ai rien eu, alors tu ne m'as rien, vraiment rien pris, alors je suis de nouveau aussi misérable que je l'étais, repoussé dans le néant, dans le néant ${ }^{6}$. 
La réponse de Tamare est sans appel. Carlotta s'est donnée à lui de plein gré. Alviano punit alors d'un coup de poignard l'égoïsme meurtrier de son rival, devenant lui-même un meurtrier narcissique.

Dans la folie, où Alviano finit par sombrer dans les toutes dernières pages de l'opéra, se manifeste la forme ultime de sa crispation sur l'image. Contrairement au miroir magique des contes de fées, les dernières paroles de Carlotta ne lui ont pas renvoyé le reflet espéré mais, au contraire, celui qu'il redoutait le plus :

Carlotta (le repoussant avec une expression de terreur et de panique effroyable)

Va-t-en! Un gnome! Un spectre nocturne ${ }^{7}$ !

N'étant pas parvenue à trouver son centre de gravité en elle-même, la personnalité d'Alviano se rend corps et âme à la régression narcissique. Son moi se fixe, cette fois de manière irréversible, sur une image - celle du fou - qui lui tiendra désormais lieu d'identité :

Alviano (la voix entièrement altérée, l'expression hagarde, comme s'il cherchait quelque chose)

Je veux, oui, où est donc ce violon. Je veux enfin, il est temps d'aller à la kermesse ! Et ma marotte, ma belle marotte, rouge, avec des grelots d'argent, personne n'a-t-il vu ma marotte? Laissez-moi, il faut enfin... (Il trébuche sur le corps de Tamare) Halte, qu'est-ce que c'est? Il y a quelqu'un, braves gens, il y a un mort.

(Il traverse en titubant la foule, qui s'écarte respectueusement, et s'éloigne vers le fond) $)^{8}$

\section{L'auto-érotisme chez Carlotta}

Mais le plus étrange était ce tableau : une main pâle et cireuse comme celle d'un mort, avec des doigts étonnamment longs et décharnés, tenant fermement quelque chose que l'on ne voyait pas. Seule une faible lueur pourpre s'échappait de ces doigts de spectre, mais cette lueur était comme une sourde plainte, comme des pleurs des gémissements contenus et comme un cri, étouffé dans les affres de la mort, appelant la délivrance ${ }^{9}$.

C'est dans la scène de l'atelier du deuxième acte, sous couvert de décrire et d'expliquer le tableau d'une amie, que Carlotta confesse à Alviano, en train de poser pour elle, la maladie cardiaque qui lui interdit l'accès au plaisir érotique. Au lieu de se tendre vers l'autre pour le toucher ou pour l'étreindre, cette «main pâle et cireuse comme celle d'un mort, avec des doigts étonnamment longs et décharnés » reste repliée en « tenant fermement quelque chose que l'on ne voyait pas » et qui n'est autre que le cœur malade de l'artiste. Les résonances narcissiques de cette allégorie sont indubitables: Carlotta met en abîme dans ce tableau sa propre incapacité à sortir de son obsession; le cœur souffrant est présenté comme le noyau de la personnalité de l'artiste, comme un véritable substitut de son identité, obligeant les mains, celles qui tiennent le pinceau, à ne se préoccuper que de lui. Le parallèle avec Alviano, l'autre stigmatisé de l'opéra, s'impose de lui-même : dans les deux cas, un handicap d'ordre génétique provoque une atrophie générale de la personnalité, une crispation d'ordre narcissique sur le défaut jugé rédhibitoire. La jeune femme le confesse d'ailleurs elle-même en termes assez clairs :

Toute la force qui soutenait sa pauvre vie était l'envie de célébrité ; et la source à laquelle elle puisait était sa souffrance non pas morale, mais physique ${ }^{10}$.

Toute la dynamique narcissique commune aux deux stigmatisés est décrite dans ces deux lignes : c'est d'une part l'«envie de célébrité » compensatrice, la même qui 
conduit Alviano à faire don de son île; et c'est d'autre part le choix de faire de leur handicap originel le moteur unique de leur création artistique, avec la conséquence perverse de figer toujours un peu plus la personnalité des deux protagonistes dans la conscience et l'expression du mal dont ils sont victimes. La jeune femme s'identifie à sa maladie cardiaque de même qu'Alviano fait de sa laideur le « cœur » de son identité.

Le narcissisme de Carlotta, comme celui de son alter ego masculin, est donc très largement imputable à la nature et bénéficie, si l'on se situe sur un plan moral, de "circonstances atténuantes" qu'il serait hors de propos de nier. Mais tout aussi indéniable est le choix que fait Carlotta, comme l'a fait Alviano, de laisser sa tendance narcissique prendre l'ascendant sur sa propre dynamique d'évolution. Si cette tendance demeure assez anodine lorsqu'elle n'engage que la relation de l'artiste à son œuvre, elle prend en revanche une orientation nettement plus dangereuse quand elle met en jeu la relation à l'autre. En tombant amoureuse de son modèle, comme nous le montre la suite de la scène de l'atelier, Carlotta est, de fait, victime d'une illusion narcissique dont les conséquences vont s'avérer proprement dévastatrices. Ce qu'elle aime dans Alviano, c'est uniquement le regard, ce regard où elle lit la passion que sa propre beauté éveille chez son modèle et qui manque encore à son tableau. C'est la quête doublement esthétique de ce regard (regard fixé sur elle et regard à fixer sur la toile) qui amène peu à peu Carlotta à quitter le ton de la conversation mondaine, à se rapprocher du badinage amoureux et pour finir à faire sa déclaration d'amour. L'effet qu'elle produit sur Alviano est puissant et immédiat ; Carlotta en profite pour se précipiter, non dans les bras de son modèle, mais sur son chevalet:

Mon Dieu, Signor, quels yeux vous faites! Prenez mes mains et embrassez-les vite!

Mais laissez-moi à mon travail ! J'ai besoin de vos yeux pour mon tableau... comme

cela ils me conviennent ${ }^{11}$.

Il s'en faut de peu que la scène ne s'achève sur l'union charnelle d'Alviano et Carlotta. C'est parce qu'il a compris le mal dont souffre Carlotta (ce qui, au passage, n'exclut pas l'argument du manque d'expérience) qu'Alviano résiste à l'invitation tacite de la jeune femme à s'unir à lui. En réalité, le fait que Carlotta soit prête à risquer sa vie en se donnant à Alviano ne plaide pas non plus pour la profondeur et la véracité de ses sentiments ; au contraire, cette velléité d'abandon au couple éros/thanatos relève de la pulsion aveugle et ne tient aucun compte de la personnalité réelle de l'être aimé. C'est, dissimulée derrière l'effervescence amoureuse, la dynamique narcissique que l'on voit ici continuer sur sa lancée, avant de s'arrêter définitivement avec l'entrée de la servante. La réalité, que cette dernière réintroduit dans l'atelier, va alors rapidement dissiper les restes du mirage amoureux de Carlotta.

Dès sa première apparition au troisième acte, alors qu'elle est officieusement fiancée à Alviano, Carlotta avoue sans ambages au duc Adorno que son amour pour Alviano a pris fin avec l'achèvement du tableau :

[...] mais depuis que j'ai terminé le tableau qui vous a tant plu, le portrait d'Alviano, c'est comme si, en moi, quelque chose s'était épuisé, comme si mon amour n'était plus le même. Non pas appauvri - comprenez-moi bien - mais comme s'il m'avait donné tout ce qu'il pouvait donner et comme si je n'avais plus rien, absolument plus rien à attendre de lui ${ }^{12}$.

La beauté et la sensualité de l'île s'emparent peu à peu de Carlotta, ravivant le désir érotique que la retenue d'Alviano, à la fin de l'acte précédent, a laissé inassouvi. Son fiancé n'étant pas parvenu à combler le manque, par définition sans fond, qu'éprouve 
son moi narcissique, ce dernier se fixe à présent sur un autre objet, Tamare, dont elle avait pourtant repoussé les avances au premier acte.

Avec le recul, on peut penser que cette évolution brutale et paradoxale de Carlotta n'est pas seulement imputable aux sortilèges de l'Élysée. Le narcissisme latent de la fille du Podestat a, en effet, révélé toute son ampleur dans la scène de l'atelier, aboutissant à un effacement progressif de la réalité au profit de la projection subjective. Portée par une exaltation esthétique fortement teintée de narcissisme, Carlotta s'est construit un Alviano « sur mesures » destiné à recueillir son fantasme d'absolu. Une fois l'exaltation retombée et le fantasme dissipé - du fait de l'achèvement du tableau - Carlotta rejette l'objet, désormais inutile à ses yeux. La fantasmagorie érotique de l'île d'Alviano a donc surtout pour effet d'amplifier et d'accélérer les manifestations du syndrome narcissique chez Carlotta. La sublimation du désir charnel par l'œuvre d'art ayant montré ses limites, c'est l'abandon à la pulsion érotique qui apparait à Carlotta comme le moyen unique d'affranchir son moi insatiable des limites qui lui sont imposées.

Il n'y a donc, sur le fond, pas vraiment lieu de faire une distinction entre les sentiments éprouvés par Carlotta pour Alviano à l'acte II et pour Tamare à l'acte III. Les deux hommes font fonction de miroirs magiques permettant à la jeune femme de connaître des satisfactions d'ordre narcissique. La différence n'est même pas qualitative, puisque Carlotta était déjà prête, à la fin de la scène de l'atelier, à s'abandonner à Alviano et, par là même, à sa pulsion mortelle. En regard de la passion narcissique qui habite Carlotta, les avantages naturels dont bénéficie Tamare sur son rival sont assez secondaires. Après avoir aimé l'âme de l'un, elle tombe amoureuse du corps de l'autre, sans que l'attirance physique soit un facteur déterminant. Si elle se tourne à présent résolument vers Tamare c'est d'une part qu'elle le sait capable de franchir l'interdit devant lequel Alviano, sous l'effet de son surmoi, a abdiqué. Mais on peut penser, d'autre part, que Carlotta n'est pas non plus totalement insensible au prestige du séducteur. Carlotta aime badiner, elle aime plaire (comme en témoigne sa première rencontre avec Tamare) et ce trait mondain de sa personnalité se manifeste sur lîle avec d'autant plus de force que les beaux et nobles sentiments qu'elle croyait éprouver pour l'âme d'Alviano se sont volatilisés. Désormais revenue de cette illusion humaniste, elle se souvient du récit que le duc Adorno lui a fait «de l'amour malheureux du beau et fougueux Tamare » et le trouve "attirant»: le regard du jeune Don Juan génois lui apparaît encore plus flatteur et désirable du fait que ce regard est lui-même désiré ou jugé désirable par d'autres.

41 Détail révélateur, les retrouvailles des amants donneront lieu à bien peu d'échanges, aussi peu qu'à l'acte I. Le fait que les deux protagonistes portent des masques à ce moment-là confirme que l'un comme l'autre ne porte aucun intérêt véritable à la personnalité qui lui fait face. Tamare tient enfin dans ses mains l'objet de son désir, tandis que Carlotta peut enfin s'enivrer du regard brûlant qui perce à travers le masque, comme si lui seul importait :

Vous m'aimez? Tant mieux. Mais laissez-moi voir à travers votre masque si vos yeux sont clairs ou sombres. Alviano ? Non, vous ne l'êtes pas. De belle stature, un joli garçon ${ }^{13}$ !

La réplique, si l'on y regarde de plus près, est un parfait condensé des amours narcissiques de Carlotta. Une première condition doit être remplie : Carlotta doit être aimée pour pouvoir aimer elle-même, ce dont elle a pu s'assurer au premier acte en ce qui concerna Tamare et au second en ce qui concerne Alviano. Le masque qui ne laisse 
voir que les yeux - qu'on imagine brûlants de désir - parachève le tableau d'un amour qui ne s'intéresse à l'autre qu'en tant que miroir. Quand ensuite Carlotta évoque la différence physique entre les deux rivaux, c'est moins pour vanter la beauté propre de Tamare que pour s'assurer que celui-ci ne la décevra pas : il a les yeux clairs et non sombres, ce qui signifie métaphoriquement que son désir n'est pas contrarié par les scrupules et les tourments propres à la personnalité «sombre » d'Alviano. Enfin, le caractère impersonnel et stéréotypé de l'hommage rendu par Carlotta au physique avantageux de son futur amant: «De belle stature, un joli garçon!» suggère qu'il lui est, au fond, indifférent de savoir qui se trouve sous le masque, pourvu qu'il s'agisse d'un bel homme, amoureux d'elle et décidé à aller jusqu'au bout de sa conquête.

La passation de pouvoir entre Alviano et Tamare est alors prononcée par Carlotta en des termes métaphoriques dont elle seule possède la clef :

Carlotta (d'une voix entièrement changée)

[...] Donne-moi ta main, ah, elle est douce et brûlante et agréable à tenir ; ici sens-tu mon cœur, comme il bat à se rompre? Pour toi, mon doux ami! Mais écoute-moi, jeune garçon, avec tes mains, si souples et si tendres, tu le tiens avec douceur et tu ne dois pas le torturer ; tu le tiens dans tes mains, doucement et sans bruit. Hop là, mon doux $\mathrm{ami}^{14}$ !

On reconnaît l'univers pictural de la jeune femme: l'obsession des mains comme symbole du contact et de l'élan vers l'autre, et, bien sûr, le cœur malade que la main de Tamare est invitée à ménager. À travers ces propos, Carlotta place son partenaire exactement dans la même situation qu'Alviano au second acte, après qu'il a découvert le tableau caché ${ }^{15}$. Le passage fait aussi écho à l'admonestation adressée à Alviano au moment de donner ses derniers coups de pinceau: «Pourtant, tu dois être bon avec moi, Alviano, (respirant avec une difficulté maladive) et tendre, mon amour ! Car je suis un jouet bien fragile ${ }^{16}$. » La peur de la mort se manifeste donc à nouveau chez Carlotta à l'approche de l'union physique, mais la fin de la déclaration : « Hop là, mon doux ami !» et le bref duo qui suit, alors que les deux amants se dirigent vers la grotte, ne laissent aucun doute sur l'issue du combat qui va s'engager, à l'intérieur de la jeune femme, entre l'instinct de conservation et l'abandon au narcissisme de mort.

\section{Tamare ou le moi surpuissant}

Dès sa première apparition, Tamare est présenté comme le chef de la clique des aristocrates débauchés dont la première scène de l'opéra nous a révélé les agissements. Contrairement à Alviano et Carlotta, tous deux habités de sentiments et de désirs ambivalents, le comte Andrae Vitelozzo Tamare est un personnage monolithique qui ne connaît pas plus le doute que le scrupule. Toute sa psychologie repose in fine sur les avantages dont la nature ainsi que l'existence l'ont largement gratifié : il est jeune, beau, riche, puissant; il est aussi aimé et admiré. La conscience de soi et l'égoïsme atteignent chez cet enfant gâté des sommets proprement vertigineux dont on ne trouve guère d'équivalent dans le monde de l'opéra.

Tamare fait lui-même, dans son entretien avec le duc Adorno au deuxième acte, la description de son mode de vie quasi somnambulique.

Il y a des hommes, ô Duc, qui ne voient que la lumière et à qui les ténèbres sont étrangères. J'étais l'un d'entre eux. La vie me semblait être une source de joie à laquelle je buvais à grands traits ; insouciant, sans réfléchir. Je tendais la main, cueillais la rose, inspirais son parfum et arrachais ses pétales. Si je me laissais 
tomber, je tombais sur un lit de mousse; les épines s'écartaient sur mon passage ainsi que les pierres qui eussent pu me blesser. Si un ami se plaignait de ses maux, de ses douleurs, je l'écoutais, mais ne le comprenais pas ${ }^{17}$. que lui inspirent les autres victimes des enlèvements. Pas plus que ces dernières Carlotta n'accède, à ses yeux, au statut de sujet. Elle demeure un pur objet de consommation dont il espère tirer toutes les jouissances possibles, au besoin par le mariage et, s'il le faut, par le viol.

51 Cette menace physique lancée par Tamare pourrait sonner comme une simple bravade si l'opéra ne nous avait pas déjà informés sur les rapts suivis d'orgies perpétrés par sa clique. L'hyperbole utilisée par Schreker permet donc de mesurer jusqu'où la pulsion narcissique peut entraîner ceux qui ont commencé à y succomber. Tamare le confesse lui-même au duc Adorno qui lui demande, au deuxième acte, de mettre fin à cette débauche organisée: il est difficile, explique-t-il, «d'endiguer la passion, une fois qu'elle est déchaîné ${ }^{19}$ ». Propos qui trouveront une parfaite illustration au troisième acte: alors que l'Élysée vient d'être ouvert au public et que leur secret peut être découvert à tout moment, Tamare et ses amis - sans doute aussi excités par le danger continuent à célébrer leurs orgies dans la grotte. donc jeter une lumière vive, quasi aveuglante, à la fois sur la personnalité de Tamare et sur l'état d'une société et d'une civilisation qui rendent de tels agissements possibles. C'est ce qui explique, au passage, que la figure de Tamare relève moins de la psychologie que de la typologie. À travers cette caricature de débauché, digne du 
Marquis de Sade, Schreker semble consciemment ou non pointer le danger de déshumanisation qui guette toute société tentée par la voie du narcissisme consumériste.

53 Mais cette dimension sociocritique n'est-elle pas également inscrite dans les profils psychologiques des deux autres héros des Stigmatisés? Le Disneyland crée par Alviano et l'effet qu'il produit sur Tamare et sa clique, sur Carlotta, mais aussi sur le peuple illustrent parfaitement le profit et le pouvoir que l'on peut tirer d'un usage massif et régressif de l'image. Quand à la maladie de cœur de Carlotta et le phénomène de surévaluation, voire d'idéalisation de la pulsion sexuelle qui l'accompagne, on serait tenté d'y voir la métaphore d'une société d'individus dominés par le Ça et incapables d'engager une relation d'amour authentique et constructive avec l'Autre. De ce point de vue, les trois protagonistes du drame et les trois invraisemblances qui leur sont associées brossent le portrait désastreux d'une société qui s'est détournée des principes civilisateurs et qui, obéissant à une logique de régression typiquement narcissique, s'est donné l'apparence, la pulsion et la violence comme horizon indépassable. Il est clair que la Renaissance où Schreker a choisi de situer son opéra fait un peu plus qu'anticiper l'évolution des sociétés dites « avancées » au xxe siècle.

\section{NOTES}

1. Le résumé et les traductions sont tirés du livret accompagnant la version CD des Stigmatisés par Lothar Zagrosek, parue chez Decca, coll. Entartete Musik, 1994.

2. „Morgen Abend, nach Einbruch der Dämm'rung, im Beisein des hohen Senates und des Podestà, mit großem Gepräng geb' ich's preis allem Volk. Ihr Herren, ihr seid geladen.“

3. Alviano, [...] Doch ich war mir bewußt einer Schuld, eines frevlen Gedankens, der Früchte zeitigte, die ich verdammte und deren Gift an der Seele mir fraß. Diese Schuld zu sühnen, hab' ich das Liebste, das damals noch meinem Herrzen lebte, das einzige Glück meiner freudlosen Tage geopfert, ohn' daß ich's bereu', denn unverdient hoher Lohn ist mir geworden.“

4. Alviano (wie gehetzt) „Ihre Stimme hör' ich! Sie schallt mir aus Höh'n, und Klüften, vertausendfacht! Gesang aus den Lüften, ein Spottlied aus Tiefen der Hölle! Ihre Gestalt tanzt vor mir, lugt aus Gebüschen und Hecken, da und dort glitzert ihr Kleid, wie ein Irrwisch hetzt sie mich, bergauf und talab, in den Äther, in Sümpfe, und ich hielt sie doch, sie war ja doch mein! Oder war sie's nicht?"

5. „Aber da innen tief bliebe ja doch so etwas, versteh' mich, wie ein weher Trost.“

6. „Doch wenn du mir sagst: ,Sie hat sich mir gegeben, sie, Carlotta, freiwillig, in Liebe und sie war glücklich' - Ja, wahrhaftig, du sagtest ,glücklich' - ja, dann, dann, dann hab' ich ja nichts gehabt, dann hast du mir ja nichts genommen, dann bin ich ja wieder ganz so elend, wie ich war, zurückgestoßen ins Nichts, ins Nichts."

7. Carlotta (ihn von sich stoßend, mit dem Ausdruck höchsten Grauens, furchtbarster Angst) „Fort, fort! Ein Alb! Ein Nachtgesicht!"

8. Alviano (mit ganz veränderter Stimme und irrem Ausdruck, als ob er etwas suchte) „Ich will, ich will, ja wo ist nur die Fiedel. Ich muß ja doch endlich zur Kirchweih! Und meine Kappe, meine schöne Kappe, rot und mit silbernen Schellen, sah niemand die Kappe? Laßt mich, ich muß ja doch 
endlich... (Er stolpert über Tamares Leichnam) Halt, was war das? Da liegt ja einer, ihr guten Leute, da liegt ja ein Toter." (Er taumelt durch die Menge dem Hintergrund zu; alles macht ihm scheu Platz) 9. „Doch das Seltsame war ein Bild: Eine Hand bleich und wächsern, wie die eines Toten, mit unheimlich langen, dürren Fingern, hielt ein Etwas umkrampft, was man nicht sah. Nur ein schwach purpurn Leuchten sickerte durch die gespenstischen Finger, doch dieser Schein war wie stumme Klage, wie unterdrücktes wimmerndes Weinen und wie ein Schrei, verhalten und todesbang, wie ein verhaltener Schrei nach Erlösung.“

10. „Die treibende Kraft dieses armen Lebens war Gier nach Ruhm; und die Quelle, aus der es schöpfte war Leid, nicht seelisch, ein körperlich Leiden.“

11. „Um Gott, Signor, was macht ihr für Augen! Da habt meine Hände und küßt sie rasch! Doch laßt mich zur Arbeit! Ich brauch' eure Augen zu meinem Bilde... so sind sie mir recht.“

12. „[...] und doch, seit ich das Bild vollendet, das euch so sehr gefiel, das Bild Alvianos, ist mir, als wär' da innen etwas erschlafft, als wär' meine Liebe nicht mehr dieselbe. Nicht ärmer versteht mich - doch als hätt' er mir nun sein Alles, sein Höchstes gegeben und ich nichts mehr, gar nichts mehr zu erwarten."

13. „Ihr liebt mich? 'S ist gut. Doch laßt mich blicken durch eure Maske, ob hell sind eure Augen oder trübe. Seid ihr Alviano? Nein, ihr seid's nicht. Schön von Gestalt, ein schmucker Knabe!“

14. Carlotta (mit ganz veränderter Stimme) „Seltsam, mein Liebling? Gib deine Hand, ah, sie ist weich und heiß, und wohlig fühlt sie sich an; da, spürst du mein Herz, wie es heftig schlägt? Dir entgegen, mein Süßer! Doch, höre, Knabe, mit deinen Händen, so weich und linde, hältst du es sanft, und darfst's nicht quälen; hältst es umspannt, ganz lind und leise. Hoppla, mein Süßer!“

15. Et que Carlotta, presque évanouie, a sans doute dévoilé sous la dictée inconsciente de son instinct de survie.

16. „Doch du mußt gut zu mir sein, Alviano, (schwer, krankhaft schwer atmend) und zart mein Liebster! Denn ich bin ein gar gebrechliches Spielzeug."

17. „Es gibt Menschen, Herzog, die sehen nur Licht, und das Dunkel ist ihnen fremd. Solch einer war ich. Das Leben schien mir ein Born der Freude, aus dem ich trank mit durstigen Zügen; sorglos, ohn' Besinnen. Reckt'ich die Hand aus, hielt ich die Rose, sog ihren Duft und zerwühlte die Blüte. Ließ ich mich fallen, fiel ich auf Moos; mich mieden die Dornen und spitzen Steine. Klagte ein Freund mir von Leid und Schmerzen, hört ich ihn wohl, doch verstand ihn nicht.“

18. „Höre, Herzog, ich will sie vergessen, ich will (langsam) sie vergessen... (mit höchstem Nachdruck) Doch erst bis sie mein ward. Bis ihres Mundes Odem ich in mich getrunken; bis diese Hände getränkt sind vom Duft ihres Haares, bis diese Frau, will sie mein Weib nicht sein, meine Dirne ward!"

19. „wie schwer einzudämmen Leidenschaft, einmal entfesselt.“

\section{RÉSUMÉS}

L'étude proposée part du contraste saisissant entre la facture classicisante de l'opéra de Schreker (progression régulière de l'intrigue selon le schéma exposition / péripétie / catastrophe, structure équilibrée des trois actes, respect de l'unité d'action, cadre rassurant de la Renaissance italienne) et la psychologie hors norme des principaux personnages. Pour entrer dans le livret et par conséquent dans l'univers des Stigmatisés, le lecteur/auditeur doit accepter certaines données plus ou moins invraisemblables, sous peine de rester définitivement à l'extérieur du drame. Nous 
essaierons de montrer que, loin d'être une faiblesse, ce déséquilibre est pleinement assumé par Schreker. Les invraisemblances et les outrances du livret ne sont qu'un moyen de montrer jusqu'où peut aller la pulsion destructrice et autodestructrice de la psyché humaine quand celleci n'est plus irriguée par la relation authentique à l'Autre. Les Stigmatisés méritent plus que tout autre le qualificatif d'«opéra limite »; c'est l'opéra de l'enfermement narcissique à l'ère de l'individualisme moderne.

Der scharfe Kontrast zwischen dem klassizistischen Aufbau der Oper (regelmäßiges Fortschreiten der Handlung nach dem Schema Exposition/Peripetie/Katastrophe, ausgewogene Struktur der drei Akte, Einheit der Handlung, italienische Renaissance als historischer Hintergrund) und der abnormen Psychologie der drei Hauptfiguren ist der Ausgangspunkt der folgenden Studie. Um sich in das Libretto und somit in die Welt der Gezeichneten hineinzuversetzen, muss der Leser bzw. Zuschauer gewisse, kaum glaubwürdige Gegebenheiten akzeptieren, wenn er sich dem Drama nicht völlig verschließen will. Eigentlich verrät die betreffende Diskrepanz keine dramatische Inkonsequenz; sie gehört vielmehr zur tieferen Bedeutung der Oper. Die unglaubwürdigen Extravaganzen des Librettos dienen nur als Vergrößerungsglas, um zu zeigen, wie zerstörerisch und selbstzerstörerisch die menschliche Psyche sein kann, wenn kein gesundes, echtes Verhältnis zum Andern sie ernährt. Durch die Schilderung von mehreren Grenzerfahrungen zeigen Schrekers Gezeichnete wie keine andere Oper die Allmacht des narzisstischen Triebs im Zeitalter des modernen Individualismus.

\section{INDEX}

Mots-clés : opéra limite

oeuvrecitee Les Stigmatisés

\section{AUTEURS}

\section{ALAIN LEDUC}

Université du Littoral-Côte d'Opale 